\title{
Refinements of some $2 D$ nonlinear integral inequalities and applications in fractional integral equations
}

\author{
K. Boukerrioua ${ }^{1 *}$, D. Diabi ${ }^{2}$ and B. Kilani ${ }^{3}$
}

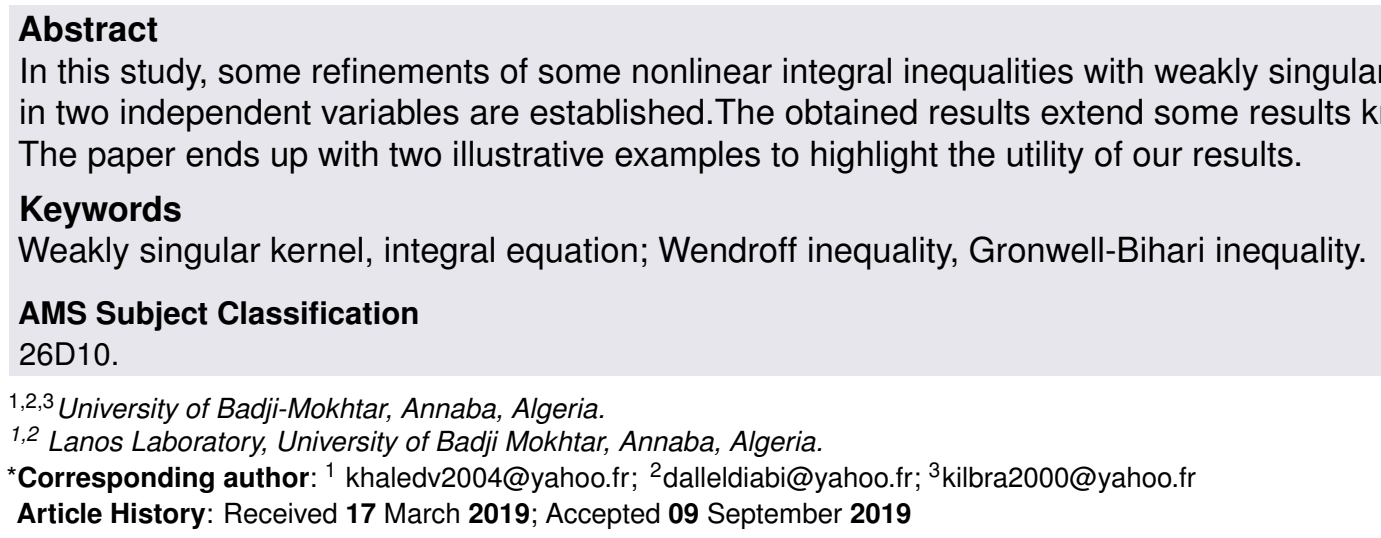

\section{Contents}

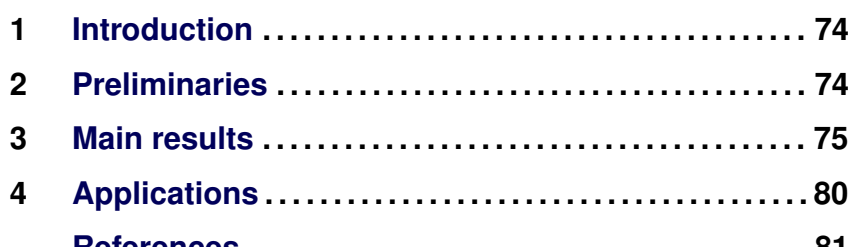

\section{Introduction}

Integral inequalities play a dominant role in the study quantitative properties of solutions of differential and integral equations. One of the most famous inequalities of this type is known as "Gronwall's inequality", "Bellman's inequality," or "Gronwall-Bellman's inequality"see $[1,2,3,8,9,12]$. Recently, the celebrated Gronwall inequality and its generalizations play increasingly important roles in the qualitative analysis of differential, integral and integro-differential equations. These Gronwall -Bellman type inequalities established have proved to be useful in the research of boundedness, uniqueness, and continuous dependence of solutions to differential and integral equations as well as difference equations. In the book [4], D. Henry proposed a method to estimate solutions of linear integral inequality with weakly singular kernel. His inequality plays the same role in the geometric theory of parabolic partial differential equations (see $[4,11])$ as the well known Gronwall inequality in the theory of ordinary differential equations. Ye et al. [5] proved a generalization of this type of inequalities and used it to study the dependence of the solution on the order and the initial condition of a fractional differential equation.

In this paper, we study a certain class of nonlinear weakly singular integral inequalities of Wendroff Type which extend some known weakly singular inequalities for functions in two variables and can be used in the analysis of various problems in the theory of certain classes of integral equations and evolution equations.

\section{Preliminaries}

Now in this section we give some basic Lemmas which are used in our subsequent discussions.

In the following, $\mathbb{R}$ denotes the set of real numbers, $\mathbb{N}$ denotes the set of integer numbers, $\mathbb{R}_{+}=[0, \infty[, \alpha, \beta>0$, $p \geq q>0, p \geq r>0$ are constants, $D:=(x, y): 0 \leq x<T$, $0 \leq y<T\}=[0, T[\times .[0, T[(0<T \leq \infty)$.

Lemma 2.1. [7] Assume that $a \geq 0, p \geq q \geq 0$ and $p \neq 0$ then

$$
a^{\frac{q}{p}} \leq \frac{q}{p} k^{\frac{q-p}{p}} a+\frac{p-q}{p} k^{\frac{q}{p}}
$$


for any $k>0$.

Lemma 2.2. (discrete Jensen inequality). Let $n \in \mathbb{N}$, $a_{1}, a_{2}, \ldots, a_{n}$ be nonnegative real numbers.Then, for $r>1$,

$$
\left(a_{1}+a_{2}+\ldots a_{n}\right)^{r} \leq n^{r-1}\left(a_{1}^{r}+a_{2}^{r}+\ldots a_{a}^{r}\right) .
$$

Lemma 2.3. (see [9, page 329]) Let $u(x, y), p(x, y), q(x, y)$ and $k(x, y)$ be nonnegative continuous functions defined for $x, y \in \mathbb{R}_{+}$. If

$$
u(x, y) \leq p(x, y)+q(x, y) \int_{0}^{x} \int_{0}^{y} k(s, t) u(s, t) d s d t
$$

for $x, y \in \mathbb{R}_{+}$, then

$$
\begin{aligned}
u(x, y) \leq & p(x, y)+q(x, y)\left(\int_{0}^{x} \int_{0}^{y} k(s, t) p(s, t) d s d t\right) \\
& \times \exp \left(\int_{0}^{x} \int_{0}^{y} k(s, t) q(s, t) d s d t\right)
\end{aligned}
$$

Furthermore, if $p(x, y)$ is nondecreasing, then we have

$$
u(x, y) \leq p(x, y) \exp \left(\int_{y_{0}}^{y} \int_{x_{0}}^{x} q(s, t) k(s, t) d s d t\right) .
$$

\section{Main results}

Theorem 3.1. Let $u(x, y), a(x, y), h(x, y)$ be nonnegative continuous functions on $D$. Let $g: \mathbb{R}_{+} \rightarrow \mathbb{R}_{+}$is a differentiable increasing function on $] 0, \infty[$ with continuous non-increasing first derivative $g^{\prime}$ on $] 0, \infty[$. If $u(x, y)$ satisfies

$$
\begin{aligned}
u^{p}(x, y) \leq & a(x, y)+\frac{1}{\Gamma(\alpha) \Gamma(\beta)} h(x, y) \\
& \times \int_{0}^{y} \int_{0}^{x}(x-s)^{\alpha-1}(y-t)^{\beta-1} \\
& g\left(u^{q}(s, t)\right) d s d t,
\end{aligned}
$$

then

$$
u(x, y) \leq\left[a(x, y)+e^{x+y} \Psi^{\frac{1}{n}}(x, y)\right]^{\frac{1}{p}},
$$

for $(x, y) \in D$, where

$$
\begin{aligned}
\Psi(x, y)= & w(x, y)+2^{n-1} L^{n} h^{n}(x, y) \\
& \times\left(\int_{0}^{y} \int_{0}^{x} \bar{A}^{n}(s, t) w(s, t) d s d t\right) \\
& \times \exp \left(2^{n-1} L^{n} \int_{0}^{y} \int_{0}^{x} \bar{A}^{n}(s, t)\right. \\
& \left.h^{n}(s, t) d s d t\right), \\
\widetilde{a}(x, y)= & \frac{1}{\Gamma(\alpha) \Gamma(\beta)} h(x, y) \\
& \times \int_{0}^{y} \int_{0}^{x}(x-s)^{\alpha-1}(y-t)^{\beta-1} \\
& g\left(\frac{q}{p} k^{\frac{q-p}{p}} a(s, t)+\frac{p-q}{p} k^{\frac{q}{p}}\right) d s d t
\end{aligned}
$$

$$
A(x, y)=\frac{q}{p} k^{\frac{q-p}{p}} a(x, y)+\frac{p-q}{p} k^{\frac{q}{p}}
$$

$$
\left\{\begin{array}{l}
\bar{A}(x, y)=g^{\prime}(A(x, y)) \\
w(x, y)=2^{n-1} \widetilde{a}^{n}(x, y) e^{-n(x+y)}
\end{array}\right.
$$

and

$$
\begin{aligned}
L= & \frac{q}{p} k^{\frac{q-p}{p}} \frac{1}{\Gamma(\alpha) \Gamma(\beta)} \\
& \times\left(\frac{\Gamma(m \alpha-m+1) \Gamma(m \alpha-m+1)}{m^{2+m(\alpha+\beta-2)}}\right)^{\frac{1}{m}}, \\
& m \geq 1, n \geq 1, \frac{1}{m}+\frac{1}{n}=1 .
\end{aligned}
$$

Proof. Define a function $z(x, y)$ by

$$
\begin{aligned}
z(x, y)= & \frac{1}{\Gamma(\alpha) \Gamma(\beta)} h(x, y) \\
& \int_{0}^{y} \int_{0}^{x}(x-s)^{\alpha-1}(y-t)^{\beta-1} g\left(u^{q}(s, t)\right) d s d t, \\
& (x, y) \in D,
\end{aligned}
$$

then

$$
u^{p}(x, y) \leq a(x, y)+z(x, y),
$$

and

$$
u(x, y) \leq(a(x, y)+z(x, y))^{\frac{1}{p}}, \quad(x, y) \in D,
$$

$$
\begin{aligned}
z(x, y) \leq & \frac{1}{\Gamma(\alpha) \Gamma(\beta)} h(x, y) \\
& \times \int_{0}^{y} \int_{0}^{x}(x-s)^{\alpha-1}(y-t)^{\beta-1} \\
& g\left((a(s, t)+z(s, t))^{\frac{q}{p}}\right) d s d t .
\end{aligned}
$$

By virtue of Lemma 2.1,for any $k>0$,

$$
\begin{aligned}
z(x, y) \leq & \frac{1}{\Gamma(\alpha) \Gamma(\beta)} h(x, y) \int_{0}^{y} \int_{0}^{x}(x-s)^{\alpha-1}(y-t)^{\beta-1} \\
& \times g\left(\frac{q}{p} k^{\frac{q-p}{p}}(a(s, t)+z(s, t))+\frac{p-q}{p} k^{\frac{q}{p}}\right) d s d t .
\end{aligned}
$$

Applying the mean value Theorem for the function $g$, then for every $c_{1}>c_{2}>0$, there exists $\left.c \in\right] c_{2}, c_{1}[$ such that

$$
g\left(c_{1}\right)-g\left(c_{2}\right)=g^{\prime}(c)\left(c_{1}-c_{2}\right) \leq g^{\prime}\left(c_{2}\right)\left(c_{1}-c_{2}\right),
$$


then we have

$$
\begin{aligned}
z(x, y) \leq & \frac{1}{\Gamma(\alpha) \Gamma(\beta)} h(x, y) \\
& \times \int_{0}^{y} \int_{0}^{x}(x-s)^{\alpha-1}(y-t)^{\beta-1} \\
& \times\left[g\left(\frac{q}{p} k^{\frac{q-p}{p}} a(s, t)+\frac{p-q}{p} k^{\frac{q}{p}}\right)\right. \\
& +g^{\prime}\left(\frac{q}{p} k^{\frac{q-p}{p}} a(s, t)+\frac{p-q}{p} k^{\frac{q}{p}}\right) \\
& \left.\times \frac{q}{p} k^{\frac{q-p}{p}} z(s, t)\right] d s d t \\
& \leq \frac{1}{\Gamma(\alpha) \Gamma(\beta)} h(x, y) \\
& \times \int_{0}^{y} \int_{0}^{x}(x-s)^{\alpha-1}(y-t)^{\beta-1} \\
& \times g\left(\frac{q}{p} k^{\frac{q-p}{p}} a(s, t)+\frac{p-q}{p} k^{\frac{q}{p}}\right) d s d t \\
& +\frac{q}{p} k^{\frac{q-p}{p}} \frac{1}{\Gamma(\alpha) \Gamma(\beta)} h(x, y) \\
& \times \int_{0}^{y} \int_{0}^{x}(x-s)^{\alpha-1}(y-t)^{\beta-1} \\
& \times g^{\prime}\left(\frac{q}{p} k^{\frac{q-p}{p}} a(s, t)+\frac{p-q}{p} k^{\frac{q}{p}}\right) z(s, t) d s d t,
\end{aligned}
$$

the above inequality can be rewritten as

$$
\begin{aligned}
z(x, y) \leq & \widetilde{a}(x, y)+\frac{q}{p} k^{\frac{q-p}{p}} \frac{1}{\Gamma(\alpha) \Gamma(\beta)} h(x, y) \\
& \times \int_{0}^{y} \int_{0}^{x}(x-s)^{\alpha-1}(y-t)^{\beta-1} \\
& \times \bar{A}(s, t) z(s, t) d s d t,
\end{aligned}
$$

where $\widetilde{a}(x, y)$ and $\bar{A}(x, y)$ are defined as in (3.4).

The last inequality can be expressed as

$$
\begin{aligned}
z(x, y) \leq & \widetilde{a}(x, y)+\frac{q}{p} k^{\frac{q-p}{p}} \frac{1}{\Gamma(\alpha) \Gamma(\beta)} \\
& \times h(x, y) \int_{0}^{y} \int_{0}^{x}(x-s)^{\alpha-1} e^{s}(y-t)^{\beta-1} \\
& \times e^{t}\left[e^{-(s+t)} \bar{A}(s, t) z(s, t)\right] d s d t,
\end{aligned}
$$

we choose suitable indices $m, n$. Applying the Hőlder inequality with indices $m, n$ to (3.11), we get

$$
\begin{aligned}
z(x, y) \leq & \widetilde{a}(x, y)+\frac{q}{p} k^{\frac{q-p}{p}} \frac{1}{\Gamma(\alpha) \Gamma(\beta)} h(x, y) \\
& \times\left[\int_{0}^{y} \int_{0}^{x}(x-s)^{m(\alpha-1)} e^{m s}\right. \\
& \left.\times(y-t)^{m(\beta-1)} e^{m t} d s d t\right]^{\frac{1}{m}} \\
& \times\left[\int_{0}^{y} \int_{0}^{x} e^{-n(s+t)} \bar{A}^{n}(s, t)\right. \\
& \left.\times z^{n}(s, t) d s d t\right]^{\frac{1}{n}} .
\end{aligned}
$$

For the first integral in (3.12), we have the estimate:

$$
\begin{aligned}
& \int_{0}^{y} \int_{0}^{x}(x-s)^{m(\alpha-1)} e^{m s}(y-t)^{m(\beta-1)} e^{m t} d s d t \\
& =e^{m(x+y)} \int_{0}^{x} \sigma^{m(\alpha-1)} e^{-m \sigma} \int_{0}^{y} \eta^{m(\beta-1)} e^{-m \eta} d \sigma d \eta \\
& =\frac{e^{m(x+y)}}{m^{2+m(\alpha+\beta-2)}} \int_{0}^{m x} \delta^{m(\alpha-1)} e^{-\delta} \int_{0}^{m y} \zeta^{m(\beta-1)} e^{-\zeta} d \delta d \zeta \\
& =\frac{e^{m(x+y)}}{m^{2+m(\alpha+\beta-2)}} \Gamma(m \alpha-m+1) \Gamma(m \beta-m+1) .
\end{aligned}
$$

Therefore we obtain from (3.12),

$$
\begin{aligned}
z(x, y) \leq & \widetilde{a}(x, y)+\frac{q}{p} k^{\frac{q-p}{p}} \frac{1}{\Gamma(\alpha) \Gamma(\beta)} h(x, y) \\
& \times\left[\frac{e^{m(x+y)}}{m^{2+m(\alpha+\beta-2)}} \Gamma(m \alpha-m+1)\right. \\
& \times \Gamma(m \alpha-m+1)]^{\frac{1}{m}} \\
& \times\left[\int_{0}^{y} \int_{0}^{x} e^{-n(s+t)} \bar{A}^{n}(s, t) z^{n}(s, t) d s d t\right]^{\frac{1}{n}} .
\end{aligned}
$$

Which gives

$$
\begin{aligned}
z(x, y) \leq & \widetilde{a}(x, y)+\operatorname{Lh}(x, y) e^{x+y} \\
& \times\left[\int_{0}^{y} \int_{0}^{x} e^{-n(s+t)} \bar{A}^{n}(s, t) z^{n}(s, t) d s d t\right]^{\frac{1}{n}},
\end{aligned}
$$

where $L$ is defined as in (3.5),

By using Lemmas 2.2, we obtain

$$
\begin{aligned}
\left(\left(e^{-(x+y)} z(x, y)\right)^{n} \leq\right. & 2^{n-1} \widetilde{a}^{n}(x, y) e^{-n(x+y)}+ \\
& +2^{n-1} L^{n} h^{n}(x, y) \\
& \times \int_{0}^{y} \int_{0}^{x} \bar{A}^{n}(s, t) \\
& \times\left(e^{-(s+t)} z(s, t)\right)^{n} d s d t
\end{aligned}
$$

the inequality (3.14) can be rewritten as

$$
\begin{aligned}
v^{n}(x, y) \leq & w(x, y)+2^{n-1} L^{n} h^{n}(x, y) \\
& \times \int_{0}^{y} \int_{0}^{x} \bar{A}^{n}(s, t) v^{n}(s, t) d s d t,
\end{aligned}
$$

where

$$
v(x, y)=e^{-(x+y)} z(x, y), w(x, y)=2^{n-1} \widetilde{a}^{n}(x, y) e^{-n(x+y)} .
$$


By Lemma 2.3 and the last inequality, we have

$$
\begin{aligned}
v^{n}(x, y) \leq & w(x, y)+2^{n-1} L^{n} h^{n}(x, y) \\
& \times\left(\int_{0}^{y} \int_{0}^{x} \bar{A}^{n}(s, t) w(s, t) d s d t\right) \\
& \times \exp \left(2^{n-1} L^{n} \int_{0}^{y} \int_{0}^{x} \bar{A}^{n}(s, t) h^{n}(s, t) d s d t\right)
\end{aligned}
$$

Using (2.16) and (2.17), we get

$$
z(x, y) \leq e^{x+y} \Psi^{\frac{1}{n}}(x, y)
$$

where $\Psi(x, y)$ is defined as in (3.3), using (3.18), and (3.8), we obtain (3.2).

Remark 3.2. In [6], the author also discussed the inequality (3.1) given in Theorem 2.1 in the case where the function $g$ satisfy :

$$
e^{-q t}(g(u))^{q} \leq R(t) g\left(e^{-q t} u^{q}\right), \forall u \geq 0,0 \leq t<T,
$$

and in [12], the authors discussed the inequality

$$
\begin{aligned}
u^{p}(x, y) \leq & a(x, y)+\frac{1}{\Gamma(\alpha) \Gamma(\beta)} h(x, y) \\
& \times \int_{0}^{y} \int_{0}^{x}(x-s)^{\alpha-1}(y-t)^{\beta-1} \\
& \times L(s, t, u(s, t)) d s d t
\end{aligned}
$$

where

$$
0 \leq L(s, t, u)-L(s, t, v) \leq T(u-v) .
$$

Corollary 3.3. Assume that the hypotheses of Theorem 2.1 hold.If

$$
\begin{aligned}
u^{p}(x, y) \leq & a(x, y)+\frac{1}{\Gamma(\alpha) \Gamma(\beta)} h(x, y) \\
& \times \int_{0}^{y} \int_{0}^{x}(x-s)^{\alpha-1}(y-t)^{\beta-1} \\
& \times \arctan \left(u^{q}(s, t)\right) d s d t,(x, y) \in D,
\end{aligned}
$$

then we have

$$
u(x, y) \leq\left[a(x, y)+e^{x+y} \varphi^{\frac{1}{n}}(x, y)\right]^{\frac{1}{p}}, \quad(x, y) \in D
$$

where

$$
\begin{aligned}
\varphi(x, y)= & w(x, y)+2^{n-1} L^{n} h^{n}(x, y) \\
& \times\left(\int_{0}^{y} \int_{0}^{x} \bar{A}^{n}(s, t) w(x, y)\right) \\
& \times \exp \left(2^{n-1} L^{n} \int_{0}^{y} \int_{0}^{x} \bar{A}^{n}(s, t) h^{n}(x, y)\right) d s d t
\end{aligned}
$$

$$
\begin{aligned}
& w(x, y)=2^{n-1} \widetilde{a}^{n}(x, y) e^{-n(x+y)} \\
& \widetilde{a}(x, y)=\frac{1}{\Gamma(\alpha) \Gamma(\beta)} h(x, y) \int_{0}^{y} \int_{0}^{x}(x-s)^{\alpha-1}(y-t)^{\beta-1} \\
& \arctan \left(\frac{q}{p} k^{\frac{q-p}{p}} a(s, t)+\frac{p-q}{p} k^{\frac{q}{p}}\right) d s d t \\
& L=\frac{q}{p} k^{\frac{q-p}{p}} \frac{1}{\Gamma(\alpha) \Gamma(\beta)} \\
& \times\left(\frac{\Gamma(m \alpha-m+1) \Gamma(m \alpha-m+1)}{m^{2+m}(\alpha+\beta-2)}\right)^{\frac{1}{m}} \\
& \bar{A}(s, t)=\frac{1}{1+\left(\frac{q}{p} k^{\frac{q-p}{p}} a(s, t)+\frac{p-q}{p} k^{\frac{q}{p}}\right)^{2}}
\end{aligned}
$$

Theorem 3.4. Let $u(x, y), a(x, y), h(x, y), b(x, y)$ be nonnegative continuous functions on D.Let $g: \mathbb{R}_{+} \rightarrow \mathbb{R}_{+}$is a differentiable increasing function on $] 0, \infty[$ with continuous nonincreasing first derivative $g^{\prime}$ on $] 0, \infty[$. If $u(x, y)$ satisfies

$$
\begin{aligned}
u^{p}(x, y) \leq & a(x, y)+\int_{0}^{y} \int_{0}^{x} b(s, t) u^{q}(s, t) d s d t+ \\
& +\frac{1}{\Gamma(\alpha) \Gamma(\beta)} \\
& \times h(x, y) \int_{0}^{y} \int_{0}^{x}(x-s)^{\alpha-1}(y-t)^{\beta-1} \\
& g\left(u^{q}(s, t)\right) d s d t
\end{aligned}
$$

then we have

$$
u(x, y) \leq\left[a(x, y)+e^{x+y} \Psi_{1}^{\frac{1}{n}}(x, y)\right]^{\frac{1}{p}}
$$

where

$$
\begin{aligned}
\Psi_{1}(x, y)= & w(x, y)+2^{n-1} L_{1} \widehat{h}^{n}(x, y) \\
& \times\left(\int_{0}^{y} \int_{0}^{x} \bar{A}^{n}(s, t) w(x, y)\right) \\
& \times \exp \left(2^{n-1} L_{1} \int_{0}^{y} \int_{0}^{x} \bar{A}^{n}(s, t)\right. \\
& \left.\widehat{h}^{n}(x, y)\right) d s d t
\end{aligned}
$$

$$
\begin{aligned}
\widehat{a}_{1}(x, y)= & \frac{q}{P} k^{\frac{q-p}{p}} \int_{0}^{y} \int_{0}^{x} b(s, t) a(s, t)+ \\
& +\frac{P-q}{P} K^{\frac{q}{p}} \int_{0}^{y} \int_{0}^{x} b(s, t) d s d t+ \\
& +\frac{1}{\Gamma(\alpha) \Gamma(\beta)} h(x, y) \times \\
& \times \int_{0}^{y} \int_{0}^{x}(x-s)^{\alpha-1}(y-t)^{\beta-1} \\
& g\left(\frac{q}{p} k^{\frac{q-p}{p}} a(s, t)+\frac{p-q}{p} k^{\frac{q}{p}}\right) d s d t
\end{aligned}
$$




$$
\begin{gathered}
\left.\widehat{h}_{1}(x, y)=\exp \left(\frac{q}{p} k^{\frac{q-p}{p}} \int_{0}^{y} \int_{0}^{x} b(s, t) d s d t\right)\right) h(x, y), \\
A(s, t)=g^{\prime}\left(\frac{q}{p} k^{\frac{q-p}{p}} a(s, t)+\frac{p-q}{p} k^{\frac{q}{p}}\right) \\
L_{1}=\frac{q}{p} k^{\frac{q-p}{p}} \frac{1}{\Gamma(\alpha) \Gamma(\beta)}\left(\frac{\Gamma(m \alpha-m+1) \Gamma(m \alpha-m+1)}{m^{2+m(\alpha+\beta-2)}}\right)^{\frac{1}{m}} \\
\frac{1}{n}+\frac{1}{m}=1 .
\end{gathered}
$$

Proof. Define a function $v(x, y)$ by

$$
\begin{aligned}
v(x, y)= & \int_{0}^{y} \int_{0}^{x} b(s, t) u^{q}(s, t) d s d t+\frac{1}{\Gamma(\alpha) \Gamma(\beta)} h(x, y) \\
& \times \int_{0}^{y} \int_{0}^{x}(x-s)^{\alpha-1}(y-t)^{\beta-1} g\left(u^{q}(s, t)\right) d s d t
\end{aligned}
$$

then

$$
u^{p}(x, y) \leq a(x, y)+v(x, y),(x, y) \in D,
$$

and

$$
\begin{aligned}
u(x, y) \leq & {[a(x, y)+z(x, y)]^{\frac{1}{p}} } \\
v(x, y) \leq & \int_{0}^{y} \int_{0}^{x} b(s, t)\left((a(s, t)+v(s, t))^{\frac{q}{p}}\right) d s d t \\
& +\frac{1}{\Gamma(\alpha) \Gamma(\beta)} h(x, y) \\
& \times \int_{0}^{y} \int_{0}^{x}(x-s)^{\alpha-1}(y-t)^{\beta-1} \\
& \times g\left[(a(s, t)+z(s, t))^{\frac{q}{p}}\right] d s d t .
\end{aligned}
$$

Using Lemma 2.1, we obtain that

$$
\begin{aligned}
v(x, y) \leq & \int_{0}^{y} \int_{0}^{x} b(s, t) \\
& \times\left[\frac{q}{p} k^{\frac{q-p}{p}}(a(s, t)+v(s, t))+\right. \\
& \left.+\frac{p-q}{p} K^{\frac{q}{P}}\right] d s d t+\frac{1}{\Gamma(\alpha) \Gamma(\beta)} \times \\
& \times h(x, y) \int_{0}^{y} \int_{0}^{x}(x-s)^{\alpha-1}(y-t)^{\beta-1} \times \\
& \times\left[g \left(\frac{q}{p} k^{\frac{q-p}{p}}(a(s, t)+v(s, t))+\right.\right. \\
& \left.\left.+\frac{p-q}{p} k^{\frac{q}{p}}\right)\right] d s d t .
\end{aligned}
$$

Applying the mean value Theorem for the function $g$, then for every $c_{1}>c_{2}>0$, there exists $\left.c \in\right] c_{2}, c_{1}$ [such that

$$
g\left(c_{1}\right)-g\left(c_{2}\right)=g^{\prime}(c)\left(c_{1}-c_{2}\right) \leq g^{\prime}(y)\left(c_{1}-c_{2}\right),
$$

then, $v(x, y)$ can be estimated as

$$
\begin{aligned}
v(x, y) \leq & \int_{0}^{y} \int_{0}^{x} b(s, t)\left[\frac{q}{P} k^{\frac{q-p}{p}}(a(s, t)+v(s, t))+\right. \\
& \left.+\frac{p-q}{p} K^{\frac{q}{p}}\right] d s d+\frac{1}{\Gamma(\alpha) \Gamma(\beta)} h(x, y) \\
& \times \int_{0}^{y} \int_{0}^{x}(x-s)^{\alpha-1}(y-t)^{\beta-1} \\
& \times\left[g\left(\frac{q}{p} k^{\frac{q-p}{p}} a(s, t)+\frac{p-q}{p} k^{\frac{q}{p}}\right)\right. \\
& +g^{\prime}\left(\frac{q}{p} k^{\frac{q-p}{p}} a(s, t)+\frac{p-q}{p} k^{\frac{q}{p}}\right) \\
& \left.\times\left(\frac{q}{p} k^{\frac{q-p}{p}} v(s, t)\right)\right] d s d t
\end{aligned}
$$

$$
\begin{aligned}
v(x, y) \leq & \int_{0}^{y} \int_{0}^{x} b(s, t)\left[\frac{q}{p} k^{\frac{q-p}{p}}(a(s, t)+v(s, t))\right. \\
& \left.+\frac{p-q}{p} K^{\frac{q}{p}}\right] d s d t \\
& +\frac{1}{\Gamma(\alpha) \Gamma(\beta)} h(x, y) \\
& \times \int_{0}^{y} \int_{0}^{x}(x-s)^{\alpha-1}(y-t)^{\beta-1} \\
& \times g\left(\frac{q}{p} k^{\frac{q-p}{p}} a(s, t)+\frac{p-q}{p} k^{\frac{q}{p}}\right) d s d t \\
& +\frac{q}{p} k^{\frac{q-p}{p}} \frac{1}{\Gamma(\alpha) \Gamma(\beta)} h(x, y) \\
& \times \int_{0}^{y} \int_{0}^{x}(x-s)^{\alpha-1}(y-t)^{\beta-1} \\
& \times g^{\prime}\left(\frac{q}{p} k^{\frac{q-p}{p}} a(s, t)+\frac{p-q}{p} k^{\frac{q}{p}}\right) v(s, t) d s d t
\end{aligned}
$$

Let

$$
\begin{aligned}
z(x, y)= & \frac{q}{p} k^{\frac{q-p}{p}} \int_{0}^{y} \int_{0}^{x} b(s, t) a(s, t) d s d t \\
& +\frac{p-q}{p} K^{\frac{q}{p}} \int_{0}^{y} \int_{0}^{x} b(s, t) d s d t \\
& +\frac{1}{\Gamma(\alpha) \Gamma(\beta)} h(x, y) \times \\
& \times \int_{0}^{y} \int_{0}^{x}(x-s)^{\alpha-1}(y-t)^{\beta-1} \\
& g\left(\frac{q}{p} k^{\frac{q-p}{p}} a(s, t)+\frac{p-q}{p} k^{\frac{q}{p}}\right) d s d t \\
& +\frac{q}{p} k^{\frac{q-p}{p}} \frac{1}{\Gamma(\alpha) \Gamma(\beta)} h(x, y) \times \\
& \times \int_{0}^{y} \int_{0}^{x}(x-s)^{\alpha-1}(y-t)^{\beta-1} \\
& \times g^{\prime}\left(\frac{q}{p} k^{\frac{q-p}{p}} a(s, t)+\frac{p-q}{p} k^{\frac{q}{p}}\right) v(s, t) d s d t .
\end{aligned}
$$


Then we have

$$
\begin{aligned}
v(x, y) & \leq z(x, y)+\frac{q}{P} k^{\frac{q-p}{p}} \times \\
& \times \int_{0}^{y} \int_{0}^{x} b(s, t) v(s, t) d s d t,(x, y) \in D .
\end{aligned}
$$

Remarking that $z(x, y)$ is nondecreasing, applying lemma 2.3 (with $\left.q(x, y)=\frac{q}{P} k^{\frac{q-p}{p}}\right)$ to $(2.28)$, we get

$$
\begin{aligned}
v(x, y) & \leq z(x, y) \\
& \exp \left(\frac{q}{p} k^{\frac{q-p}{p}} \int_{0}^{y} \int_{0}^{x} b(s, t) d s d t\right)(x, y) \in D .
\end{aligned}
$$

Moreover,

$$
\begin{aligned}
& z(x, y) \leq \frac{q}{p} k^{\frac{q-p}{p}} \int_{0}^{y} \int_{0}^{x} b(s, t) a(s, t) d s d t \\
& +\frac{p-q}{p} K^{\frac{q}{P}} \int_{0}^{y} \int_{0}^{x} b(s, t) d s d t \\
& +\frac{1}{\Gamma(\alpha) \Gamma(\beta)} h(x, y) \times \\
& \times \int_{0}^{y} \int_{0}^{x}(x-s)^{\alpha-1}(y-t)^{\beta-1} \\
& \times g\left(\frac{q}{p} k^{\frac{q-p}{p}} a(s, t)+\frac{p-q}{p} k^{\frac{q}{p}}\right) d s d t \\
& +\frac{q}{p} k^{\frac{q-p}{p}} \frac{1}{\Gamma(\alpha) \Gamma(\beta)} h(x, y) \\
& \times \int_{0}^{y} \int_{0}^{x}(x-s)^{\alpha-1}(y-t)^{\beta-1} \\
& \times g^{\prime}\left(\frac{q}{p} k^{\frac{q-p}{p}} a(s, t)+\frac{p-q}{p} k^{\frac{q}{p}}\right) \\
& \times z(s, t) \exp \left(\frac{q}{p} k^{\frac{q-p}{p}}\right. \\
& \left.\int_{0}^{t} \int_{0}^{s} b(\tau, \xi) d \tau d \xi\right) d s d t, \\
& z(x, y) \leq \frac{q}{p} k^{\frac{q-p}{p}} \int_{0}^{y} \int_{0}^{x} b(s, t) a(s, t) d s d t \\
& +\frac{p-q}{p} K^{q} \int_{0}^{y} \int_{0}^{x} b(s, t) d s d t+ \\
& +\frac{1}{\Gamma(\alpha) \Gamma(\beta)} h(x, y) \times \\
& \times \int_{0}^{y} \int_{0}^{x}(x-s)^{\alpha-1}(y-t)^{\beta-1} \\
& \times g\left(\frac{q}{p} k^{\frac{q-p}{p}} a(s, t)+\frac{p-q}{p} k^{\frac{q}{p}}\right) d s d t+ \\
& +\frac{q}{p} k^{\frac{q-p}{p}} \frac{1}{\Gamma(\alpha) \Gamma(\beta)} \times \\
& \times \exp \left(\frac{q}{p} k^{\frac{q-p}{p}} \int_{0}^{y} \int_{0}^{x} b(s, t) d s d t\right) \\
& \times h(x, y) \int_{0}^{y} \int_{0}^{x}(x-s)^{\alpha-1}(y-t)^{\beta-1} \\
& \times g^{\prime}\left(\frac{q}{p} k^{\frac{q-p}{p}} a(s, t)+\frac{p-q}{p} k^{\frac{q}{p}}\right) z(s, t) d s d t
\end{aligned}
$$

$$
\begin{aligned}
z(x, y) \leq & \widehat{a}_{1}(x, y)+\frac{q}{p} k^{\frac{q-p}{p}} \frac{1}{\Gamma(\alpha) \Gamma(\beta)} \\
& \times \widehat{h}_{1}(x, y) \int_{0}^{y} \int_{0}^{x}(x-s)^{\alpha-1}(y-t)^{\beta-1} \\
& \times g^{\prime}\left(\frac{q}{p} k^{\frac{q-p}{p}} a(s, t)+\frac{p-q}{p} k^{\frac{q}{p}}\right) z(s, t) d s d t \\
& =\widehat{a}_{1}(x, y)+\frac{q}{p} k^{\frac{q-p}{p}} \frac{1}{\Gamma(\alpha) \Gamma(\beta)} \\
& \times \widehat{h}_{1}(x, y) \int_{0}^{y} \int_{0}^{x}(x-s)^{\alpha-1}(y-t)^{\beta-1} \\
& \times \bar{A}_{1}(s, t) \times z(s, t) d s d t
\end{aligned}
$$

where $\widehat{a}_{1}(x, y)$ and $\widehat{h}_{1}(x, y), \bar{A}_{1}(x, y)$ are defined as in (3.23).

The inequality (3.30) is similar to the inequality (3.10). So following in a similar manner to the proof in Theorem 3.1, we get that

$$
z(x, y)=e^{x+y} \Psi_{1}^{\frac{1}{n}}(x, y),
$$

where

$$
\begin{aligned}
\Psi_{1}(x, y)= & w(x, y)+2^{n-1} L^{n} \widehat{h}_{1}^{n}(x, y) \\
& \times\left(\int_{0}^{y} \int_{0}^{x} \bar{A}_{1}^{n}(s, t) w(s, t)\right) \\
& \times \exp \left(2^{n-1} L_{1} \int_{0}^{y} \int_{0}^{x} \bar{A}_{1}^{n}(s, t) \widehat{h}_{1}^{n}(s, t) d s d t\right),
\end{aligned}
$$

and $L_{1}$ is defined as in (3.24).

Combining (3.23), (2.31) and (3.25), we obtain the desired result.

Remark 3.5. if we replace $g\left(u^{q}(s, t)\right.$ by $L(s, t, u(s, t))$, then Theorem 3.4 reduces to $[5$, Theorem 5$]$.

Corollary 3.6. Assume that the hypotheses of Theorem 3.4 hold. If

$$
\begin{aligned}
u^{p}(x, y) \leq & a(x, y)+\int_{0}^{y} \int_{0}^{x} b(s, t) u^{q}(s, t) d s d t \\
& +\frac{1}{\Gamma(\alpha) \Gamma(\beta)} h(x, y) \\
& \times \int_{0}^{y} \int_{0}^{x}(x-s)^{\alpha-1}(y-t)^{\beta-1} \\
& \times \log \left(1+u^{q}(s, t)\right) d s d t .
\end{aligned}
$$

Then

$$
\begin{aligned}
u(x, y)< & {\left[a(x, y)+e^{x+y} \Psi_{2}^{\frac{1}{n}}(x, y)\right]^{\frac{1}{p}} } \\
\Psi_{2}(x, y)= & w(x, y)+2^{n-1} L_{2} \widehat{h}_{1}^{n}(x, y) \times \\
& \times\left(\int_{0}^{y} \int_{0}^{x} \bar{A}^{n}(s, t) w(x, y)\right) \\
& \times \exp \left(2^{n-1} L_{2} \int_{0}^{y} \int_{0}^{x} \bar{A}^{n}(s, t){\widehat{h_{1}}}^{n}(x, y)\right) d s d t
\end{aligned}
$$

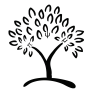




$$
\begin{aligned}
& \widehat{a}_{2}(x, y)=\frac{q}{P} k^{\frac{q-p}{p}} \int_{0}^{y} \int_{0}^{x} b(s, t) a(s, t)+ \\
& +\frac{P-q}{P} K^{\frac{q}{P}} \int_{0}^{y} \int_{0}^{x} b(s, t) d s d t \\
& +\frac{1}{\Gamma(\alpha) \Gamma(\beta)} h(x, y) \times \\
& \times \int_{0}^{y} \int_{0}^{x}(x-s)^{\alpha-1}(y-t)^{\beta-1} \\
& \times \log \left(1+\frac{q}{p} k^{\frac{q-p}{p}} a(s, t)+\frac{p-q}{p} k^{\frac{q}{p}}\right) d s d t \\
& w_{2}(x, y)=2^{n-1} \widehat{a}_{2}^{n}(x, y) e^{-n(x+y)} \\
& L_{2}=\frac{q}{p} k^{\frac{q-p}{p}} \frac{1}{\Gamma(\alpha) \Gamma(\beta)} \times \\
& \times\left(\frac{\Gamma(m \alpha-m+1) \Gamma(m \alpha-m+1)}{m^{2+m(\alpha+\beta-2)}}\right)^{\frac{1}{m}} \\
& \bar{A}(s, t)=\frac{1}{1+\frac{q}{p} k^{\frac{q-p}{p}} a(s, t)+\frac{p-q}{p} k^{\frac{q}{p}}}, \\
& \left.\widehat{h}_{2}(x, y)=\exp \left(\frac{q}{p} k^{\frac{q-p}{p}} \int_{0}^{y} \int_{0}^{x} b(s, t) d s d t\right)\right) h(x, y) .
\end{aligned}
$$

Theorem 3.7. Assume that the hypotheses of Theorem 3.4 hold. Let $g_{1}$ and $g_{2}: \mathbb{R}_{+} \rightarrow \mathbb{R}_{+}$are differentiable increasing functions on $] 0, \infty[$ with continuous non-increasing first derivative $g_{1}^{\prime}$ and $g_{2}^{\prime}$ on $] 0, \infty[$.If $u(x, y)$ satisfies

$$
\begin{aligned}
u^{p}(x, y) \leq & a(x, y)+\int_{0}^{y} \int_{0}^{x} b(s, t) g_{1}\left(u^{q}(s, t)\right) d s d t \\
& +\frac{1}{\Gamma(\alpha) \Gamma(\beta)} h(x, y) \times \\
& \times \int_{0}^{y} \int_{0}^{x}(x-s)^{\alpha-1}(y-t)^{\beta-1} \\
& g_{2}\left(u^{r}(s, t)\right) d s d t .
\end{aligned}
$$

Then

$$
u(x, y) \leq\left[a(x, y)+e^{x+y} \Psi_{3}^{\frac{1}{n}}(x, y)\right]^{\frac{1}{p}}
$$

where

$$
\begin{aligned}
\Psi_{3}(x, y)= & w_{3}(x, y)+2^{n-1} L_{3} \widehat{h}_{3}^{n}(x, y) \\
& \times\left(\int_{0}^{y} \int_{0}^{x} \bar{A}^{n}(s, t) w(x, y)\right) \\
& \times \exp \left(2^{n-1} L^{n} \int_{0}^{y} \int_{0}^{x} \bar{A}^{n}(s, t)\right. \\
& \left.\widehat{h}^{n}(x, y)\right) d s d t
\end{aligned}
$$

$$
\begin{aligned}
& \widehat{a}_{3}(x, y)=\int_{0}^{y} \int_{0}^{x} b(s, t) \times \\
& g_{1}\left(\frac{q}{p} k^{\frac{q-p}{p}} a(s, t)+\frac{p-q}{p} k^{\frac{q}{p}}\right) d s d t \\
& +\frac{1}{\Gamma(\alpha) \Gamma(\beta)} h(x, y) \times \\
& \times \int_{0}^{y} \int_{0}^{x}(x-s)^{\alpha-1}(y-t)^{\beta-1} \\
& \times g_{2}\left(\frac{r}{p} k^{\frac{r-p}{p}} a(s, t)+\frac{p-r}{p} k^{\frac{r}{p}}\right) d s d t \\
& w_{3}(x, y)=2^{n-1} \widehat{a}_{3}^{n}(x, y) e^{-n(x+y)} \\
& L_{3}=\frac{r}{p} k^{\frac{r-p}{p}} \frac{1}{\Gamma(\alpha) \Gamma(\beta)} \\
& \times\left(\frac{\Gamma(m \alpha-m+1) \Gamma(m \alpha-m+1)}{m^{2+m(\alpha+\beta-2)}}\right)^{\frac{1}{m}}, \\
& \frac{1}{n}+\frac{1}{m}=1 \text {. } \\
& A(s, t)=g_{2}\left(\frac{r}{p} k^{\frac{r-p}{p}} a(s, t)+\frac{p-r}{p} k^{\frac{r}{p}}\right), \\
& \bar{A}(s, t)=g_{2}^{\prime}\left(\frac{r}{p} k^{\frac{r-p}{p}} a(s, t)+\frac{p-r}{p} k^{\frac{r}{p}}\right), \\
& \widehat{h}_{3}(x, y) \\
& =\exp \left(\frac{q}{p} k^{\frac{q-p}{p}} \int_{0}^{y} \int_{0}^{x} b(s, t)\right. \\
& \left.\times g_{1}^{\prime}\left(\frac{q}{p} k^{\frac{q-p}{p}} a(s, t)+\frac{p-q}{p} k^{\frac{q}{p}}\right) d s d t\right) h(x, y) .
\end{aligned}
$$

Proof. The proof would run parallel to that of Theorem 3.4. We omit the details.

Remark 3.8. If $g_{1}(x)=x, r=q$, then Theorem 3.7, reduces to Theorem 3.4.

\section{Applications}

In this section, we shall illustrate how our main results can be applied to study the boundedness and uniqueness of the solution to certain fractional-integral equations.

Example 4.1. Let us consider the following fractional lintegral equation :

$$
\begin{aligned}
z^{p}(x, y)= & l(x, y)+\frac{1}{\Gamma(\alpha) \Gamma(\beta)} \int_{0}^{y} \int_{0}^{x}(x-s)(y-t) \\
& \times G(s, t, z(s, t)) d s d t
\end{aligned}
$$

for $(x, y) \in D$, where $l(x, y)$ and $G(x, y) \in C(D \times \mathbb{R}, \mathbb{R})$. 
Suppose that

$$
\begin{aligned}
|l(x, y)| & \leq a(x, y) \\
|G(x, y, u)| & \leq M(x, y) g\left(|u|^{q}\right) \\
M(x, y) & \leq h(x, y)
\end{aligned}
$$

where the functions, $a(x, y), h(x, y) p, q$ and $g$ are as in Theorem 3.1, $M$ is nonnegative continuous function on $D$ and nondecresing. If $u(x, y),(x, y) \in D$, is any solution of $(4.1)$, then by plugging (4.2) in (4.1) and applying Theorem 3.1, we obtain a bound on the solutions $u(x, y)$ of $(4.1)$.

Proposition 4.2. Assume that the functions $G$ in (4.1) satisfies the condition

$$
|G(s, t, z)|-G(s, t, \bar{z}) \leq M(s, t) g(|z-\bar{z}|),
$$

where $g$ is defined as in Theorem 3.1 such that $g(0)=0$ and $M$ is defined as in Example 4.1.Then (4.1) has at most one solution.

Proof. Let $z_{1}(x, y)$ and $z_{2}(x, y)$ be two solutions of (4.1), then

$$
\begin{aligned}
z_{1}(x, y)= & a(x, y)+\frac{1}{\Gamma(\alpha) \Gamma(\beta)} \times \\
& \times \int_{0}^{y} \int_{0}^{x}(x-s)^{\alpha-1}(y-t)^{\beta-1} \\
& \times G\left(s, t, z_{1}(s, t)\right) d s d t
\end{aligned}
$$

and

$$
\begin{aligned}
z_{2}(x, y)= & a(x, y)+\frac{1}{\Gamma(\alpha) \Gamma(\beta)} \times \\
& \times \int_{0}^{y} \int_{0}^{x}(x-s)^{\alpha-1}(y-t)^{\beta-1} \times \\
& \times G\left(s, t, z_{2}(s, t)\right) d s d t
\end{aligned}
$$

From (4.4) and (4.5), we have $\left|z_{1}(x, y)-z_{2}(x, y)\right| \leq$

$$
\begin{aligned}
\leq & \frac{1}{\Gamma(\alpha) \Gamma(\beta)} \int_{0}^{y} \int_{0}^{x}(x-s)^{\alpha_{1}-1}(y-t)^{\beta_{1}-1} \\
& \times\left[G\left(s, t, z_{1}(s, t)\right)-G\left(s, t, z_{2}(s, t)\right] d s d t,\right.
\end{aligned}
$$

which implies

$$
\begin{aligned}
\left|z_{1}(x, y)-z_{2}(x, y)\right| \leq \\
\leq \quad \frac{h(x, y)}{\Gamma(\alpha) \Gamma(\beta)} \int_{0}^{y} \int_{0}^{x}(x-s)^{\alpha_{1}-1}(y-t)^{\beta_{1}-1} \\
\quad \times g\left(\left|z_{1}(s, t)-z_{2}(s, t)\right|\right) d s d t
\end{aligned}
$$

According to Theorem $3.1(p=q=1)$, we obtain that $|z(t, s)-\bar{z}(t, s)| \leq 0$, which implies $z_{1}(x, y)=z_{2}(x, y)$ for $(x, y) \in D$.

\section{References}

[1] T. H. Gronwall, Note on the derivatives with respect to a parameter of solutions of a system of differential equations, Ann. of Math, 20(1919), 292-296.

[2] R. Bellman The stability of solutions of linear differential equations, Duke Math. J., 10(1943), 643-647.

[3] K. Boukerrioua, D. Diabi and T.Chiheb, Further nonlinear integral inequalities in two independent variables on time scales and their applications, Malaya J. Mat. 5(1)(2017), 109-114.

[4] D. Henry, Geometric Theory of Semilinear Parabolic Equations, Springer-Verlag, Berlin, Heidelberg, New York, 1981.

[5] Ye, HP, Gao, JM, Ding, YS, A generalized Gronwall inequality and its application to a fractional differential equation, J. Math. Anal. Appl., 328(2007), 1075-1081.

[6] M. Medved, Nonlinear Singular Integral Inequalities for Functions in Two and $n$ Independent Variables, Journal of Inequalities and Applications, 5(3)(2000), 287-308.

[7] F.Jiang, F. Meng, Explicit bounds on some new nonlinear integral inequalities with delay, Journal of Computational and Applied Mathematics, 205(2007), 479-486.

[8] F. W. Meng, Q. H. Feng, B. Zheng, Explicit Bounds to Some New Gronwall-Bellman-Type Delay Integral Inequalities in Two Independent Variables on Time Scales, J Appl. Math., 2011(2011), Article 754350, 1-25.

[9] B. G. Pachpatte, Inequalities for Differential and Integral Equations, vol. 197 of Mathematics in Science and Engineering, Academic Press, San Diego, Calif, USA, 1998.

[10] B.G.Pachpatte, Integral inequalities of the Bihari type, Math. Inequal. Appl., 5(2002), 649-657.

[11] H. Sano and N. Kunimatsu, Modified Gronwall's inequality and its application to stabilization problem for semilinear parabolic systems, Systems Control Letters, 22(1994), 145-156.

[12] B. Zheng, New generalized $2 D$ nonlinear inequalities and applications in fractional differential-integral equations, J.Comput. Appl. Math., (2015), 235-246.

$$
\operatorname{ISSN}(\mathrm{P}): 2319-3786
$$

Malaya Journal of Matematik $\operatorname{ISSN}(\mathrm{O}): 2321-5666$

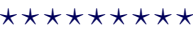

